\title{
Felder, Franziska
}

\section{Corona und die ultimativen 'memento mori'. Überlegungen zu einem besseren Umgang mit behinderungsbedingten Vulnerabilitäten in Demokratie und Bildung}

Krause, Sabine [Hrsg.]; Breinbauer, Ines Maria [Hrsg.]; Proyer, Michelle [Hrsg.]: Corona bewegt - auch die Bildungswissenschaft. Bildungswissenschaftliche Reflexionen aus Anlass einer Pandemie. Bad Heilbrunn : Verlag Julius Klinkhardt 2021, S. 153-168

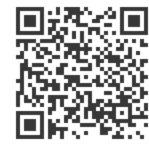

Quellenangabe/ Reference:

Felder, Franziska: Corona und die ultimativen 'memento mori'. Überlegungen zu einem besseren Umgang mit behinderungsbedingten Vulnerabilitäten in Demokratie und Bildung - In: Krause, Sabine [Hrsg.]; Breinbauer, Ines Maria [Hrsg.]; Proyer, Michelle [Hrsg.]: Corona bewegt - auch die Bildungswissenschaft. Bildungswissenschaftliche Reflexionen aus Anlass einer Pandemie. Bad Heilbrunn : Verlag Julius Klinkhardt 2021, S. 153-168 - URN: urn:nbn:de:0111-pedocs-222303 - DOI: 10.25656/01:22230

https://nbn-resolving.org/urn:nbn:de:0111-pedocs-222303

https://doi.org/10.25656/01:22230

in Kooperation mit / in cooperation with:

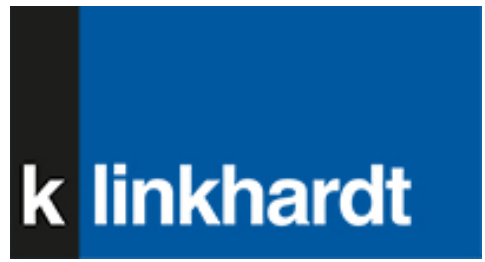

http://www.klinkhardt.de

\section{Nutzungsbedingungen}

Dieses Dokument steht unter folgender Creative Commons-Lizenz: $\mathrm{http}: / /$ creativecommons.org/licenses/by-nc-sa/4.0/deed.de - Sie dürfen das Werk bzw. den Inhalt unter folgenden Bedingungen vervielfältigen, verbreiten und öffentlich zugänglich machen sowie Abwandlungen und Bearbeitungen des Werkes bzw. Inhaltes anfertigen: Sie müssen den Namen des Autors/Rechteinhabers in der von ihm festgelegten Weise nennen. Dieses Werk bzw. der Inhalt darf nicht für kommerzielle Zwecke verwendet werden. Die neu entstandenen Werke bzw. Inhalte dürfen nur unter Verwendung von Lizenzbedingungen weitergegeben werden, die mit denen dieses Lizenzvertrages identisch oder vergleichbar sind.

Mit der Verwendung dieses Dokuments erkennen Sie die Nutzungsbedingungen an.

\section{Terms of use}

This document is published under following Creative Commons-License: http://creativecommons.org/licenses/by-nc-sa/4.0/deed.en - You may copy, distribute and transmit, adapt or exhibit the work in the public and alter, transform or change this work as long as you attribute the work in the manner specified by the author or licensor. You are not allowed to make commercial use of the work. If you alter, transform, or change this work in any way, you may distribute the resulting work only under this or a comparable license.

By using this particular document, you accept the above-stated conditions of use.

\section{Kontakt / Contact:}

peDOCS

DIPF | Leibniz-Institut für Bildungsforschung und Bildungsinformation

Informationszentrum (IZ) Bildung

E-Mail: pedocs@dipf.de

Internet: www.pedocs.de

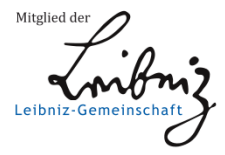




\section{Sabine Krause}

Ines Maria Breinbauer

Michelle Proyer

(Hrsg.)

\section{Corona bewegt - auch die Bildungswissenschaft}

Bildungswissenschaftliche Reflexionen aus Anlass einer Pandemie 
Krause / Breinbauer / Proyer Corona bewegt - auch die Bildungswissenschaft 


\section{Sabine Krause \\ Ines Maria Breinbauer \\ Michelle Proyer \\ (Hrsg.)}

\section{Corona bewegt - auch die Bildungswissenschaft}

Bildungswissenschaftliche Reflexionen aus Anlass einer Pandemie 
Die Veröffentlichung der Open Access-Publikation wurde durch Mittel der Universität Innsbruck und der Universität Wien möglich.

Dieser Titel wurde in das Programm des Verlages mittels eines Peer-Review-Verfahrens aufgenommen. Für weitere Informationen siehe www.klinkhardt.de.

Bibliografische Information der Deutschen Nationalbibliothek Die Deutsche Nationalbibliothek verzeichnet diese Publikation in der Deutschen Nationalbibliografie; detaillierte bibliografische Daten sind im Internet abrufbar über http://dnb.d-nb.de.

2021.h. () by Julius Klinkhardt.

Foto Umschlagseite 1: () Sabine Krause, Innsbruck.

Druck und Bindung: AZ Druck und Datentechnik, Kempten.

Printed in Germany 2021.

Gedruckt auf chlorfrei gebleichtem alterungsbeständigem Papier.

(c) (i)(5) Die Publikation (mit Ausnahme aller Fotos, Grafiken und Abbildungen) ist veröffent-

licht unter der Creative Commons-Lizenz: CC BY-NC-SA 4.0 International

https://creativecommons.org/licenses/by-nc-sa/4.0/

ISBN 978-3-7815-5889-2 digital

doi.org/10.35468/5889

ISBN 978-3-7815-2453-8 


\section{Inhalt}

Ines M. Breinbauer, Sabine Krause und Michelle Proyer

Corona bewegt - nicht nur, aber auch die Bildungswissenschaft

Barbara Platzer

Verantwortung in der Pandemie. Über die Unzulänglichkeit individueller

Verantwortung und blinde Flecken des Pandemie-Diskurses

Thorsten Fuchs und Dominik Matzinger

Lost in Pandemic. Zur Frage von adoleszenten Bildungsprozessen und

Übergängen in Zeiten des Social Distancing

Ines M. Breinbauer

Lernen an den Grenzen unseres Wissens

\section{Dominic Lüthi}

,Corona' im Ethikunterricht

Sabine Krause

Ver(un)sichern in Zeiten der Ver(un)sicherung

Andreas Köpfer

,Distant Bodies - Collective Spaces - Borders': Herstellung und

Aneignung von Lernräumen in Zeiten erforderlicher Distanzierung

Johannes Reitinger und Michelle Proyer

Studentisches Lernen im digitalen Raum in Zeiten der Pandemie

Tina Obermayr, Seyda Subasi Singh, Gertraud Kremsner,

Oliver Koenig und Michelle Proyer

Revisiting vulnerabilities - Auswirkungen der Pandemie auf die

$(\mathrm{Re})$ Konstruktion von Vulnerabilität*en im Kontext von Bildung

Franziska Felder

Corona und die ultimativen ,memento mori‘. Überlegungen zu einem besseren Umgang mit behinderungsbedingten Vulnerabilitäten in Demokratie und Bildung

Autor*innenspiegel 
Franziska Felder

\section{Corona und die ultimativen, memento mori' - Überlegungen zu einem besseren Umgang mit behinderungsbedingtenVulnerabilitäten in Demokratie und Bildung}

\section{Einleitung}

Covid-19 - so kann man mitten in der globalen Pandemie-Krise, die im Frühjahr 2020 ihren Anfang nahm, bereits konstatieren - hat die Verletzlichkeit der Gattung Mensch für Krankheiten und Behinderungen mit aller Kraft ins Bewusstsein der Öffentlichkeit gebracht. Seit Beginn der Krise bis Anfang Januar 2021 sind laut der WHO rund 83 Millionen Menschen am neuartigen Virus erkrankt und über 1.8 Million gestorben - 47\% davon auf den beiden amerikanischen Kontinenten und 32\% in Europa (World Health Organization, 5. Januar 2021). Insbesondere ältere Menschen sowie Menschen mit Vorerkrankungen und Behinderungen sind gefährdet, schwer an Covid-19 zu erkranken oder sogar daran zu sterben. In einem Beitrag in der Zürcher Zeitung Tages Anzeiger bezeichnete der Schweizer Schriftsteller Christoph Keller, der selbst an der Muskelkrankheit Spinale Muskelatrophie (SMA) erkrankt ist, Menschen mit Behinderungen auch als „ultimative Risikogruppe“ (Keller 2020, o.A.). Menschen mit Behinderungen seien ein „Memento Mori“, ein Zeichen für Verletzlichkeit, Gebrechlichkeit und Sterblichkeit. Und: Der Schutz von Menschen mit Behinderungen sei zwar dringlich, werde aber oft vergessen.

Und tatsächlich kann man konstatieren, dass Menschen mit Behinderungen in dieser Krise einer ganzen Reihe von Risiken ausgesetzt sind, die in der breiten öffentlichen Berichterstattung über die Pandemie wie auch in politischen Debatten über deren Management und die Folgen vergleichsweise wenig Beachtung finden. So listet die Weltgesundheitsorganisation (WHO) in ihrer Mitteilung über die Situation behinderter Menschen (2020) zwar eine Reihe von Herausforderungen auf, mit denen Menschen mit Behinderungen konfrontiert sind. Darunter fallen Hindernisse in der Umsetzung grundlegender Hygienemaßnahmen, beispielsweise, wenn Waschbecken unzugänglich sind oder Menschen aus motorischen Gründen Mühe haben, sich die Hände zu reiben und so Seife oder 
Desinfektionsmittel wirksam zu verteilen. Auch Schwierigkeiten bei der Einhaltung von physischen Distanzvorschriften werden als Problem für Menschen mit Behinderungen genannt. Dies ist beispielsweise dann der Fall, wenn ein Mensch mit Behinderung zusätzliche Unterstützung durch eine Assistentin oder einen Assistenten benötigt; wenn es für ihn aus sensorischen Gründen notwendig ist, Gegenstände zu berühren, um an Informationen zu gelangen; oder wenn Hindernisse im Zugang zu Informationen über aktuelle Maßnahmen im Zuge der Pandemie existieren, beispielsweise, weil diese nicht in leichter Sprache verfügbar, in Gebärdensprache übersetzt oder in Brailleschrift erhältlich sind. Die WHO erwähnt darüber hinaus auch das erhöhte Risiko für Menschen mit bestimmten gesundheitlichen Einschränkungen und Vorerkrankungen (beispielsweise Asthma oder Stoffwechselkrankheiten wie zystische Fibrose), schwer an Covid-19 zu erkranken, und zwar einerseits aufgrund des zugrundeliegenden Gesundheitszustands, andererseits aber auch aufgrund der Hindernisse im Zugang zu Gesundheitsvorsorge und grundlegenden Schutzmaßnahmen.

Die von der WHO erwähnten Vulnerabilitäten bleiben allerdings fast ausschließlich auf der Ebene von (direkten) gesundheitlichen Vulnerabilitäten und (indirekten) Herausforderungen ihrer Bewältigung respektive der Möglichkeiten, sich selbst davor schützen zu können, stehen. Nicht in den Blick geraten Vulnerabilitäten, die mit den institutionalisierten Lebensumständen zu tun haben, in denen viele Menschen mit Behinderungen leben. Auch geraten jene Risiken nicht in den Blick, die Folgen der Maßnahmen sein können, die Menschen mit Behinderungen selbst ergreifen, um sich vor den gesundheitlichen Risiken einer Ansteckung mit Covid-19 zu schützen. Die beiden letztgenannten Vulnerabilitäten basieren anders als die von der WHO angeführten nämlich weitgehend auf extern ausgelösten, häufig spezifisch Menschen mit Behinderungen (sowie auch ältere, pflegebedürftige Menschen) betreffenden Faktoren. ${ }^{1}$ Und es sind solche Risiken, die auch die weitergehende Gefährdung in sich tragen, von gesundheitlichen Aspekten auf andere Facetten menschlichen Lebens - beispielsweise sozialen - überzugreifen.

Um diese Überlegungen zu vertiefen und zu veranschaulichen, möchte ich im Folgenden verschiedene Konzeptualisierungen vorschlagen, die sowohl die von der WHO erwähnten als auch die von mir ergänzten konkreten Vulnerabilitäten theoretisch systematisieren, zusammenführen und gerade auch für die von der WHO nicht thematisierten, letztgenannten Risiken praktisch verdeutlichen. Darüber hinaus sollen in einem zweiten Teil auch Überlegungen getätigt werden, wie mit diesen Vulnerabilitäten gerade in Bildungskontexten wie der Schule in einer

1 Es sind also nicht solche, die aktuell mehr oder minder alle gleichermaßen betreffen, beispielsweise Hygiene- und Distanzvorschriften, inklusive der entsprechenden Risiken an Covid-19 zu erkranken, wenn man sie nicht einhält. 
Demokratie, die auch den Anspruch nach Partizipation aller Bürger*innen hat, besser umgegangen werden könnte. Diese Gedanken, die sich an John Deweys Überlegungen zum Zusammenhang von Demokratie und Bildung anlehnen, sind aus Platzgründen relativ schematisch und kurz. Sie verdeutlichen aber die Notwendigkeit - so die Hoffnung - Überlegungen zu pandemiebedingten Vulnerabilitäten konsequent mit Fragen nach ihrer gesamtgesellschaftlichen Thematisierung, Linderung oder Beseitigung zu verbinden. Ein kurzes Fazit, in welchem die wichtigsten Punkte nochmals zusammengefasst und kontextualisiert werden, schließt den Beitrag ab.

\section{Behinderung und Vulnerabilität}

Es wurde bereits angedeutet: Eine Reihe der Vulnerabilitäten, mit denen Menschen mit Behinderungen in der aktuellen Pandemie zu kämpfen haben, treffen auf sie als Gruppe besonders zu, sei dies in qualitativer oder in quantitativer Hinsicht. So leben viele Menschen mit Behinderungen in Institutionen, und es sind denn auch diese institutionalisierten Lebensumstände, welche einige der Risiken, an Covid-19 zu erkranken, für die Gruppe der Menschen mit Behinderungen verstärken. So ist es für diejenigen Menschen mit Behinderungen, die in einer Werkstatt oder einer Einrichtung der Behindertenhilfe arbeiten, schwierig, wenn nicht gar unmöglich, im sogenannten Homeoffice zu arbeiten. Menschen, die Therapien in Anspruch nehmen müssen, die nicht ambulant oder digital verfügbar sind, riskieren sekundäre, nicht ursächlich mit dem Covid-19 Virus in Verbindung stehende Gesundheitsprobleme (wie eine Verschlechterung ihres ursprünglichen Gesundheitszustands) oder einen Bruch oder zumindest eine Diskontinuität in den Bemühungen, eine Verbesserung der Funktionalität körperlicher Strukturen, Funktionen oder des psychischen Wohlergehens zu erreichen. Letzteres ist für diejenigen Menschen ein großes Problem, die bereits mit psychisch bedingten Einschränkungen leben. Ihre Gefährdung liegt nicht nur darin, dass Bemühungen zum Erlangen psychischen Wohlergehens zunichte gemacht werden (beispielsweise, weil Therapien nicht fortgesetzt werden können oder in den digitalen, tendenziell unpersönlichen Raum verfrachtet werden), sondern auch darin, dass die aktuelle Gesundheitsbedrohung Ängste, depressive oder anderweitig psychisch belastende Lebensphasen und Situationen verstärken kann. Menschen, die in einem Wohnheim zusammen mit anderen Menschen mit Behinderungen leben, haben Schwierigkeiten, die Forderung nach sozialem Rückzug zu erfüllen, oder aber sie werden aus Sicherheitsgründen zusätzlich sozial abgeschottet, wenn Besuche und Kontakte nach außen ausbleiben müssen. Damit steigt ihr Risiko, unter psychischen Begleiterscheinungen wie Depressionen oder Angstzuständen zu lei- 
den. Diese und weitere Risiken für Menschen mit Behinderungen sind deutlich und bekannt, auch wenn sie in der medialen Berichterstattung und der politischen Debatte aktuell kaum Beachtung finden.

Um diese Risiken aber systematisch einordnen und Vulnerabilität bei einer Behinderung in der aktuellen Pandemie umfassend verstehen zu können, ist ein ganzheitlicheres Denkmodell notwendig. Dazu ist es hilfreich, zunächst einmal Klarheit darüber zu gewinnen, was eigentlich unter Vulnerabilität verstanden werden kann. Denn - so wird sich zeigen - Vulnerabilität sollte nicht einfach als ein anthropologisches Faktum verstanden werden, welches grundsätzlich alle Menschen betrifft. Als solches wird sie in der Diskussion um die Risiken in der aktuellen Pandemie, aber auch in anderen Themenbereichen (wie allgemeinen Risiken, eine Krankheit zu haben oder einen Unfall zu erleiden) nämlich häufig verstanden. Behinderung wird dann als ein Risiko gesehen, dem jeder Mensch prinzipiell ausgesetzt ist, einfach aus dem Grund, weil die Gattung Mensch verletzlich ist. Vulnerabilität sollte demgegenüber auch als Phänomen gesehen werden, welches sich kulturell und sozial verstärkt und - auch unter Gerechtigkeitsgesichtspunkten - problematische, aber vermeidbare Formen annehmen kann. Diese Form der Vulnerabilität ist anders als die erste eben nicht biologisch an die Gattung Mensch und ihre Verletzlichkeit gebunden. Diese zweite Form der Vulnerabilität wird vielmehr erst gesellschaftlich geschaffen und verstärkt, und zwar über die Art und Weise, wie gesellschaftliche Institutionen (beispielsweise der medizinischen Versorgung oder der Bildung) funktionieren. Nicht zuletzt auch die Art und Weise, wie über Behinderung (nicht) gesprochen wird, ist ein Teil dieses größeren Gerechtigkeitsproblems, das eine sogenannte ,problematische' Vulnerabilität von Menschen mit Behinderung zum Ausdruck bringt.

\subsection{Fundamentale und Problematische Vulnerabilität}

Aber gehen wir zunächst einen Schritt zurück. In einer ersten Annäherung kann man Vulnerabilität ganz allgemein folgendermaßen definieren:

"Vulnerability results from a situation in which an agent is both dependent on and exposed to another agent. To be vulnerable is to be exposed to the power of someone we depend on - physically, affectively, socially or economically. In this respect, vulnerability supposes the existence of a relationship of dependency between agents who have the power to act on one another, and potentially, to harm one another" (Garrau \& Laborde 2015, 52).

Vulnerabilität ergibt sich also aus einer Abhängigkeit der Handlungen von jenen anderer Agenten. Sie ist relational und haftet nicht einem Menschen gewissermaßen als Zustand an. Die Definition kann attestieren, dass es existenzielle Risiken gibt, die externen Ursprungs sind. Die Covid-19-Krise ist ein solcher Fall. Allerdings beeinflussen und verstärken sich externe Risiken über die Handlungen 
unterschiedlicher Agenten, seien dies individuelle Personen, Gruppen oder Institutionen. Erst dann spricht man von Vulnerabilität. Vulnerabilität ist also kontextuell verankert und ein sozialer Tatbestand, sie ist kein (ausschließlich) biologisches Faktum. Die charakteristische Gefährdung behinderter Menschen - konkret hier ihre Covid-19 spezifische Vulnerabilität - ergibt sich also nicht nur über das Virus, seine Verbreitung und die gesundheitlichen Risiken, die damit einhergehen, sondern auch darüber, wie Menschen, Gruppen, Gesellschaften und Institutionen mit diesen pandemiebedingten Vulnerabilitäten und Risiken umgehen. Dies verdeutlicht eine zweite, nun auch auf Gerechtigkeitsüberlegungen anwendbare normative Konzeptualisierung von Vulnerabilität, die an der Stelle hilfreich ist. Der Philosoph Philip Pettit (2001) unterscheidet nämlich zwei Formen von Vulnerabilität: zum einen die, fundamentale Vulnerabilität, zum anderen die ,problematische Vulnerabilität. Beide Formen sollten nach Pettit bei Fragen einer gerechten Gesundheitsversorgung und eines gerechten Umgangs mit Krisen in einer Gesellschaft eine wichtige und gleichberechtigte Rolle spielen.

Die ,fundamentale Vulnerabilität kennzeichnet nach Pettit (ebd.) eine anthropologische Kategorie, also einen Aspekt des Menschseins. Es bedeutet: Vulnerabilität ist ein allen Menschen gemeinsames, irreduzibles Faktum menschlichen Lebens, das aus unserer wechselseitigen Abhängigkeit entsteht. Die fundamentale Vulnerabilität verdeutlicht auch, dass jeder Mensch eine bestimmte (allerdings nicht gleich verteilte ${ }^{2}$ ) statistische Wahrscheinlichkeit hat, im Verlaufe des Lebens mit einer Behinderung oder chronischen Erkrankung konfrontiert zu sein.

Sehr deutlich kommt die Bedeutung dieser fundamentalen Vulnerabilität für das Leben von Menschen in der Philosophie Alasdair MacIntyres (1985, 1988) zum Ausdruck. MacIntyre macht darin die menschliche Vulnerabilität zum Ausgangspunkt seiner ethisch-normativen Überlegungen. So schreibt er in Dependent Rational Animals (MacIntyre 1985, 1), einem seiner Hauptwerke:

"We human beings are vulnerable to many kinds of affliction and most of us are at some time afflicted by serious ills. How we cope is only in small part up to us. It is most often to others that we owe our survival, let alone our flourishing, as we encounter bodily illness and injury, inadequate nutrition, mental defect and disturbance, and human aggression and neglect. This dependence on particular others for protection and sustenance is most obvious in early childhood and in old age. But between these first and last stages our lives are characteristically marked by longer or shorter periods of injury, illness or other disablement and some among us are disabled for their entire lives."

2 So ist die Wahrscheinlichkeit im Leben eine Behinderung zu erleiden für Menschen, die sozioökonomisch benachteiligt sind, weitaus größer als für andere Menschen. Behinderung korreliert in signifikanter Weise mit Faktoren wie Armut oder mangelnder Bildung. Vgl. hierzu das 'Factsheet on Persons with Disabilities' der United Nations (2010). 
Es ist eine Erfahrung, die in der aktuellen Pandemie weite Teile der Gesellschaft machen - die Möglichkeit, nach einer Erkrankung mit Covid-19 längerfristig unter den Folgen der Viruserkrankung zu leiden. Als ein struktureller Aspekt menschlicher Existenz kann fundamentale Vulnerabilität daher weder komplett eliminiert noch aus interpersonalen Beziehungen verbannt werden (vgl. Nussbaum 2006; Tronto 2013).

Pettit (2001) nennt aber noch eine zweite Form von Vulnerabilität: die ,problematische Vulnerabilität. Diese findet in der aktuellen Pandemiebekämpfung relativ wenig Beachtung. Die ,problematische Vulnerabilität entsteht laut Pettit (ebd.) aufgrund von Dominanz- und Herrschaftsbeziehungen zwischen Menschen sowie deren praktischer Sichtbarkeit in gesellschaftlichen Hierarchien und epistemischer Macht (beispielsweise dahingehend, als glaubwürdige Zeugin wahrgenommen zu werden). Anders als die erste Form beschreibt sie die langfristigen und subjektiven Auswirkungen einer Beziehung, bei der die dominierte Person ihrer Autonomie beraubt wird, indem ihr die Art der Anerkennung verweigert wird, die Gleichheit und Respekt verlangen würde. Herrschaft oder Dominanz können, so Pettit (ebd.), Verwundbarkeiten hervorrufen, die sich in Gefühlen der Ohnmacht und Angst und schließlich in einem Mangel an Selbstachtung seitens der Dominierten manifestiert. Vulnerabilität in ihrer problematischen Dimension - als Ausdruck von Dominanz und Herrschaft - ist unter moralischen Aspekten und unter Gerechtigkeitsgesichtspunkten ein Problem. Denn Dominanz und Herrschaft drücken Ungleichheit in relationaler Hinsicht aus und verschließen gleichzeitig Freiheitsräume. Sie schaffen und verstärken also einerseits bereits bestehende Ungleichheiten und verringern andererseits die Freiheitsgrade von Menschen. Damit erhöhen ,problematische Vulnerabilitäten' auch die ,fundamentalen Vulnerabilitäten“ von Menschen (Garrau \& Laborde 2015, 56).

Vielleicht scheint es zunächst etwas übertrieben, in der Covid-19-Krise in Bezug auf Menschen mit Behinderungen auch Formen, problematischer Vulnerabilität zu vermuten. Vielmehr ist es zunächst naheliegend, hinter den eingangs genannten Risiken, die Menschen mit Behinderungen häufiger oder in anderer Form betreffen, ausschließlich Formen, fundamentaler Vulnerabilität zu vermuten. Denn was hat der schwierigere Zugang zu Gesundheitsversorgung, zu Schutzmaßnahmen (beispielsweise bezüglich der Einhaltung von Hygiene und Distanzvorschriften) mit ,problematischer Vulnerabilität' zu tun? Ja, die Vermutung eines Zusammenhangs zwischen Gesundheitskrise und ,problematischer Vulnerabilität könnte sogar sogenannten ,Corona-Skeptikern' in die Hände spielen, welche vermuten, bei all den getroffenen Maßnahmen - von Hygienevorschriften bis hin zu sogenannten Lockdowns - ginge es einzig darum, Menschen zu unterdrücken und ihrer fundamentalen zivilen Freiheiten zu berauben.

Zunächst einmal muss gesagt werden, dass viele Probleme, mit denen Menschen mit Behinderungen aktuell konfrontiert sind, tatsächlich nicht grundsätzlich 
anderer Art sind als diejenigen, welchen auch andere Menschen ausgesetzt sind. Alle Menschen fühlen sich in der Pandemie bedroht und verunsichert und müssen Einschränkungen ihrer Bewegungs- und Handlungsfreiheit in Kauf nehmen. Aber diese Freiheitseinschränkungen sind nicht per se Ausdruck von Dominanz mächtiger Personen oder Gruppen oder gesellschaftlich problematischer Hierarchien. Vielmehr sind sie erstens legitimiert durch Bezüge auf ein höheres Gut (beispielsweise Gesundheit und Wohlergehen), zweitens sind sie praktisch beschränkt (das heißt, sie beziehen sich in liberalen Gesellschaften nicht auf alle möglichen Aspekte von Freiheit, sie schränken also beispielsweise nicht ganz fundamental die Denk- oder Meinungsfreiheit ein), drittens haben sie temporären Charakter, und viertens schließlich ist es aktuell auch keineswegs eine nur Menschen mit Behinderungen betreffende Erfahrung, um die Gesundheit (die physische wie die psychische) fürchten zu müssen. Daher kann auch schlecht argumentiert werden, die Maßnahmen würden bestimmte Gruppen der Gesellschaft bewusst unterdrücken.

Qualitativ und quantitativ anders als für den Großteil der Bevölkerung aber gestalten sich für Menschen mit Behinderungen zwei Aspekte der gegenwärtigen Pandemiesituation: Erstens das Ausmaß an Herausforderungen, mit denen sie konfrontiert sind, deren institutionelle Verankerung in der Gesellschaft (die u.a. auf eine weitgehende gesellschaftliche Marginalisierung von Menschen mit Behinderungen hinweist), und in der Konsequenz auch die Maßnahmen, die Menschen mit Behinderungen oder ihre Angehören treffen müssen, um die entstandenen Schwierigkeiten oder Hürden aus dem Weg zu räumen oder deren Effekte abzumildern; zweitens die Tatsache, dass die Sichtweise und die Erfahrungen von Menschen mit Behinderungen wie auch ihre Bedürfnisse und Interessen nicht oder nur marginal Teil der öffentlichen Wahrnehmung des ,Problems' und seiner Lösung sind. Wenn Keller (2020, o.A.) von Menschen mit Behinderungen als „ultimativer Risikogruppe“ spricht, meint er nicht nur die Tatsache, dass er und andere Menschen mit Behinderungen verstärkten Gesundheitsrisiken ausgesetzt sind, sondern auch, dass diese Tatsache wenig bis gar keine gesellschaftliche Aufmerksamkeit erhält, dass gesellschaftliche Institutionen ihn und andere Menschen mit Behinderungen systematisch marginalisieren, und dass bereits bestehende Marginalisierungen sich in der Pandemie noch einmal verstärken. Bei beiden Aspekten werden zumindest Anteile ,problematischer Vulnerabilität offenbar, also Aspekte gesellschaftlicher Dominanz- und Hierarchieverhältnisse, indem man Menschen mit Behinderungen erstens viel mehr zumutet als anderen Menschen und zweitens ihre Lage wenig öffentliche (mediale wie politische) Beachtung findet.

Auf beide Punkte werde ich im Folgenden genauer eingehen. Der erste Punkt - die Art der Herausforderungen und insbesondere die Wege, die beschritten werden müssen um Risiken zu begegnen - lässt sich analysieren mit einer Kon- 
zeptualisierung, die sich im Buch Disadvantage von Jonathan Wolff und Avner De-Shalit $(2007,70)$ findet. Der zweite Hinweis, die mangelnde gesellschaftliche (kulturelle wie soziale) Aufmerksamkeit, lässt sich mit Hilfe der Theorie epistemischer Gerechtigkeit von Miranda Fricker (2007) detaillierter verstehen.

\subsection{Drei materiale Kategorien von Risiken}

Jonathan Wolff und Avner De-Shalit (2007) schlagen vor, drei Kategorien von Risiken zu unterscheiden. Ihre Kategorisierung von Risiken basiert dabei auf dem von Martha Nussbaum und Amartya Sen entwickelten Capability Ansatz (Sen 1999; Nussbaum 2006). Der Capability Ansatz kann als eine Art, Verwirklichungschancenansatz' bezeichnet werden. Mangel an Capabilities werden darin nämlich als ein Mangel an Chancen, ein gutes Leben führen zu können und eigene Vorstellungen vom Leben verwirklichen zu können, verstanden. Benachteiligung bezüglich Capabilities ist dem Ansatz nach bedingt durch eine Wechselwirkung zwischen inneren und äußeren Faktoren, also den personalen, insbesondere den körperlichen, Voraussetzungen eines Menschen und den sozialen, kulturellen und strukturellen Bedingungen der Gesellschaft, in der er lebt. Dabei wird im Capability Ansatz in besonderer Weise die Interaktion zwischen inneren und äußeren Faktoren betont, insbesondere hinsichtlich der zentralen Frage, was Menschen tun und sein können, beispielsweise, inwiefern sie personale Freiheiten also über soziale Unterstützungsmaßnahmen, Gesundheitsversorgung oder monetäre Hilfen verwirklichen können.

Die drei Kategorien von Risiken sind nach Wolff und De-Shalit (2007) folgende: Erstens sind es Risiken, die mit spezifischen Funktionen zu tun haben, beispielsweise andauernde gesundheitliche Probleme, starke Schmerzen und so weiter. Diese Risiken nennen die Autoren ,risks to specific functionings', wobei unter Funktionen vieles verstanden werden kann, nicht nur rein körperliche Zustände (beispielsweise körperliche Kraft oder Gesundheit), sondern auch Lebens- und Tätigkeitsbereiche wie Arbeit, Gesundheit, Mobilität, Bildung, Freundschaften. Covid-19 verstärkt ganz klar eine Reihe von spezifischen Risiken, nicht nur, aber auch für Menschen mit Behinderungen. Dazu gehört ganz klar das Risiko, schwer an Covid-19 zu erkranken oder gar daran zu sterben. Hierbei gibt es bestimmte Gruppen in der Gesellschaft, die ein höheres Risiko haben schwer zu erkranken, und dazu gehören auch viele Menschen mit Behinderungen, unter anderem solche, die bereits an respiratorisch bedingten Grunderkrankungen leiden.

Zweitens gibt es Risiken, die sich über bestimmte Ungleichheitskategorien hinweg bilden respektive verstärken (sogenannte, cross-category risks'). Menschen mit Behinderungen und bestimmten Vorerkrankungen sind beispielsweise in der aktuellen Pandemie dem Risiko ausgesetzt, aufgrund ihrer gesundheitlichen Einschränkungen nicht arbeiten zu können, in finanzielle Notlagen zu geraten oder aufgrund geschlossener Schulen und ausbleibender Therapien weniger gute 
Bildung zu erhalten. Damit verstärken oder erweitern sich soziale Ungleichheitskategorien.

Drittens gibt es umgekehrte, über Ungleichheitskategorien hinweggehende Risiken (sogenannte, inverse cross-category risks'). Das meint, dass jemand beim Versuch eine bestimmte Funktion abzusichern (also beispielsweise gesund zu bleiben) an anderer Stelle ein Risiko generiert. Ein Beispiel dafür ist, wenn eine Person, die sich aus Angst um ihre Gesundheit komplett aus dem Sozialleben zurückzieht, und damit tatsächlich ihre Gesundheit schützt, sich damit aber gleichzeitig und in der Konsequenz dem Risiko sozialer Isolation und Einsamkeit aussetzt. Indem diese Person also ein Risiko vermeidet, geht sie ein anderes ein. Das ist der Kern dieser dritten Form von Risiken.

Während die erste Art von Risiken in der aktuellen Pandemie alle Menschen in mehr oder minder starker Form betrifft, treffen die anderen beiden Risiken auf Menschen, die bereits ohne Pandemie unter sozialer Ungleichheit und Benachteiligungen leiden, in stärkerem Maße zu. $\mathrm{Zu}$ diesen Menschen gehören auch Menschen mit Behinderungen. Die eingangs aufgeführten Beispiele verdeutlichen dies. Wenn beispielsweise jemand in einem Wohnheim lebt, dort aufgrund der Pandemie keinen Besuch mehr empfangen kann, Ferien und Feiertage alleine verbringen muss und bei der Arbeit Distanznehmen und Schutz vor Ansteckung schwierig oder unmöglich sind, riskiert er oder sie eine Verstärkung bestimmter Ungleichheitskategorien, geht also ein , cross-category risk ein. Und wer - gerade beim Versuch, sich vor einer Ansteckung zu schützen - andere Funktionen gefährdet (beispielsweise psychische Gesundheit), erleidet ein sogenanntes ,inverse crosscategory risk: Um ihre Folgen abdämpfen und mindern zu können, wären interne wie externe Ressourcen notwendig - von monetären Ressourcen, über Beziehungen, Zugang zu Informationen, Beratung und Hilfe bis hin zu internen Ressourcen in Form von Bildung, physischer und psychischer Gesundheit und so weiter. Genau hier liegen aber sehr oft die primären Benachteiligungen von Menschen mit Behinderungen, die sich nun im Zuge der Pandemie noch zu verstärken drohen. Gerade sie, die bezüglich bestehender gesellschaftlicher, zivilgesellschaftlicher und lokaler Unterstützungsangebote an und für sich schon benachteiligt sind, riskieren in der aktuellen Lage und gerade aufgrund einer sich ständig wechselnden allgemeinen gesundheitspolitischen Risikolage - verbunden mit einem häufig eingeschränkten Tool an personalen und sozialen Ressourcen - weiterführende Benachteiligungen. So hat beispielsweise ein Mensch, der zur Bewältigung täglicher Lebensanforderungen (aufstehen, anziehen, duschen, essen, trinken etc.) persönliche Assistenz in Anspruch nehmen muss, wenig Einfluss auf das Gesundheitsverhalten seiner Assistenz, und diese wiederum hat häufig nicht denselben Zugang zu Schutzmaterial wie beispielsweise eine Altenpflegerin in einem Altersheim. Hinzu kommt, dass sich die Person mit Behinderung je nach gesundheitlicher Ausgangslage zwischen zwei schwierigen und gleichermaßen nachteiligen 
Situationen entscheiden muss: Nimmt sie die persönliche Assistenz weiterhin in Anspruch, riskiert sie eine potenzielle Ansteckung; verzichtet sie auf die persönliche Assistenz, verliert sie die notwendige Unterstützung im Alltag und riskiert vielleicht zu verwahrlosen oder soziale Kontakte zu verlieren.

Dass die Problemlagen, mit denen Menschen mit Behinderungen zu kämpfen haben, darüber hinaus vergleichsweise wenig mediale und politische Beachtung finden, hat mit einem zweiten Problem zu tun, auf das ich nun zu sprechen komme: die epistemische Ungerechtigkeit.

\subsection{Epistemische Ungerechtigkeit}

Die Tatsache, dass die spezifischen Bedürfnisse und Interessen behinderter Menschen nur marginal Teil der öffentlichen Berichterstattung und gesellschaftlicher Debatten sind, lässt sich mit Hilfe einer zweiten Konzeptualisierung analysieren. Die Philosophin Miranda Fricker spricht in ihrem Buch Epistemic Justice (2007) von zwei Formen epistemischer Ungerechtigkeit: Die eine Form nennt sie ,testimonial injustice (etwas sperrig kann man das mit, Zeugnisungerechtigkeit übersetzen); die andere und fundamentalere Form der Ungerechtigkeit nennt sie ,hermeneutical injustice' (,hermeneutische Ungerechtigkeit).

Das Bahnbrechende an dieser Systematisierung und an Frickers Buch ist dabei, dass die Autorin darin epistemische Fragen, die bis dahin nicht unter normativen Gesichtspunkten betrachtet wurden, konsequent und systematisch mit Gerechtigkeitsüberlegungen verbunden hat. Damit bezieht sie die Frage, wie wir über soziale Tatbestände reden und denken, explizit auf ihre Bedeutung für gesellschaftliche Verhältnisse. Wie sich sogleich zeigen wird, sind diese Überlegungen gerade im Hinblick auf unseren Umgang mit Menschen mit Behinderungen in der aktuellen Pandemie von großer Bedeutung.

Aber zunächst einmal zu den Grundzügen der Theorie Frickers. ,Testimonial injustice' zeichnet sich dadurch aus, dass die Aussagen und Sichtweisen von Menschen aufgrund ihrer Zugehörigkeit zu einer bestimmten sozialen Gruppe abgewertet oder ignoriert werden. Sie bezieht sich auf soziale und kulturelle Formen der Devaluation von Erfahrungen behinderter Menschen und ist gut beobachtbar an der Art und Weise, wie das Leben mit Behinderung in Kultur und Medien thematisiert wird. Das zeigt sich in der aktuellen Krise mit besonderer Deutlichkeit: Viel mediale Aufmerksamkeit erhalten Lobbygruppen, Impfskeptiker*innen, sogenannte Coronaleugner*innen, Wissenschaftler*innen und Politiker*innen sowie - schon marginaler - verschiedene Altersgruppen, von jungen Menschen bis hin zu älteren Menschen respektive Senior*innen. Praktisch absent oder ignoriert von der gesellschaftlichen und medialen Aufmerksamkeit aber sind Menschen mit Behinderungen. Aktivist*innen wie Raul Krauthausen beispielsweise weisen genau auf diese Form der Ungerechtigkeit immer wieder hin: Seine*ihre Erfahrungen sind nicht Teil der medialen Berichterstattung und sie scheinen 
auch nicht Teil politischer Überlegungen zu einem gerechten Umgang mit der Covid-19-Pandemie zu sein. Diese ,Stille' hat auch eminente materiale Folgen für Menschen mit Behinderungen, denn die Mittel und die Unterstützung, die sie benötigen würden, um mit der aktuellen Situation und ihren gemachten Erfahrungen umgehen zu können, geraten ebenfalls nicht in den Blick. Letzteres gilt insbesondere für Menschen mit psychischen oder kognitiven Einschränkungen. Noch deutlicher wird das bei der zweiten Form epistemischer Ungerechtigkeit, die gleichzeitig auch die fundamentalere ist. Die, hermeneutical injustice verletzt Menschen dadurch, dass Aspekte ihrer Identität unterdrückt werden. ,Hermeneutical injustice $e^{6}$ zeichnet sich dadurch aus, dass für die Erfahrungen von Menschen gar keine angemessene Sprache existiert. Ein Beispiel außerhalb des Themenbereichs Behinderung verdeutlicht dies. So war häusliche oder sexuelle Gewalt gegen Frauen erst wirklich als solche benennbar, als ihr Status in Bezug auf die Eherechte geändert und auch in den Medien und in der öffentlichen Wahrnehmung individuelle Erfahrungen nicht mehr einzig als persönliches Schicksal, sondern als kulturelle und soziale Muster gesehen wurden. Solange diese Bezugsrahmen fehlten, wurden die Erfahrungen betroffener Frauen häufig als bedauernswerte Einzelfälle und ihr Leiden als übertriebenes Jammern abgetan. Dasselbe gilt heute auch für Erfahrungen von Polizeigewalt, der viele schwarze Menschen in den USA ausgesetzt sind. Auch diese Form der Gewalt wird im Zuge der Black Lives Matter Bewegung erst im großen, öffentlichen Maßstab als das gesehen, was sie natürlich auch vorher material schon war: eine Ungerechtigkeit. Aber erst durch die Aufhebung hermeneutischer Schranken und eine breite öffentliche Thematisierung (z.B. in den Medien und in der Politik) wurde sie - nicht zuletzt auch für die Betroffenen selbst - als Teil einer strukturellen Ungerechtigkeit benennbar, und nicht nur als die eines individuellen Unglücks.

,Hermeneutical injustice verweist auch auf das, was Menschen von sich selbst verstehen und wissen können, beziehungsweise auf den Sinn, den sie ihren Erfahrungen über Sprache verleihen können. Das Spezifische an hermeneutischer Ungerechtigkeit besteht laut Fricker (2007) darin, dass bedeutende Anteile der individuellen sozialen Erfahrungen vom kollektiven Verständnis verdrängt werden, was wiederum eine strukturelle Ungerechtigkeit darstellt. Fundamental ist diese Form der Ungerechtigkeit, weil Betroffene keine Sprache dafür finden (können), was ihnen geschieht, und in der Folge kein oder zumindest kein detailliertes (und vor allem kein sozial geteiltes) Wissen darüber existiert, um mit diesen Erfahrungen auf einer strukturellen Ebene umzugehen.

Nun scheint es in der aktuellen Pandemie nicht so zu sein, dass generell keine Sprache dafür existieren würde für das, was Menschen widerfährt. Nur scheint diese Sprache eher mit Schicksal denn mit Gerechtigkeit in Verbindung gebracht zu werden. Gerechtigkeitsfragen tauchen allenfalls da auf, wo sie sich mit Verteilungsfragen verbinden: also beispielsweise mit Fragen einer gerechten medi- 
zinischen Versorgung bei einer Infektion (z.B. im Zugang zu Beatmungsgeräten oder Intensivpflegebetten). Individuelle soziale Erfahrungen im Umgang mit der Pandemie aber - beispielsweise dahingehend, was das Ausbleiben von Therapien, beengte Wohnverhältnisse, mangelnde soziale Kontakte und starke Einschränkungen im gewohnten Tagesablauf für ein Kind mit sich regressiv entwickelnder Stoffwechselerkrankung (die auch von Verhaltensauffälligkeiten und kognitiven Einschränkungen geprägt ist) und seine Familie bedeutet - bleiben weitgehend aus (vgl. Schulz 2020).

Ungerechtigkeit umfasst nach Fricker (2007) häufig beide epistemischen Formen, zum einen die Erfahrung, nicht als Zeug*in für bestimmte Einsichten angesehen werden, und zum anderen die hermeneutische Dimension, nicht über die angemessene Sprache, Ausdrucksmöglichkeit oder Wissenskategorien zu verfügen. Das ist nicht nur ein individuelles Problem für die Betroffenen, deren Erfahrungen nicht geglaubt wird oder die keine angemessen Sprache dafür finden, ihren Erfahrungen Ausdruck zu verleihen. Vielmehr verweisen beide Formen auf ein ethisch-moralisches Problem gesellschaftlichen Ausmaßes.

Das zeigt sich an der aktuellen Krise besonders deutlich. Das weitgehende Fehlen der Perspektiven und Stimmen behinderter Menschen im öffentlichen und politischen Diskurs über Covid-19 lässt vermuten, dass hier Formen hermeneutischer und testimonialer Marginalisierung vorliegen. Ziehen wir darüber hinaus die erste Systematik von Wolff und De-Shalit (2007) heran, dann ist auch zu befürchten, dass der Gesellschaft als Ganzer die spezifischen Risiken, denen Menschen mit Behinderungen ausgesetzt sind - insbesondere die, cross-category-risks' und die , inverse cross-category-risks ${ }^{\prime}$ - nicht bewusst sind (und dass vielleicht selbst die Betroffenen ihre Erlebnisse nicht als Ungerechtigkeiten wahrnehmen). Eine Sensibilisierung und ein Wissen um diese spezifischen Risikolagen wären aber eine Vorbedingung dafür, gesellschaftlich einen besseren und vor allem auch gerechteren Umgang mit Krisen zu erreichen.

Beide Theorien - sowohl die von Wolff und De-Shalit (2007) als auch die von Fricker (2007) - verweisen also auf die Leerstellen, die aufgrund der weitgehenden Stille, die gesellschaftlich mit dem Thema Behinderung verbunden ist, entstehen können. Menschen mit Behinderungen werden so - auch aufgrund ihrer gesellschaftlichen Marginalisierung - die Möglichkeiten genommen, ihren eigenen Erfahrungen Sinn zu verleihen, sie in den gesellschaftlichen Diskurs zu bringen und auch die notwendige gesellschaftliche Unterstützung zu erfahren. Das erleben nicht zuletzt im Moment viele Familien mit Kindern mit Behinderungen, denen vom einen Tag auf den anderen in einem sogenannten Lockdown die notwendige therapeutische oder pädagogische Unterstützung für ihre Kinder, aber auch Entlastungs- und Unterstützungsmöglichkeiten für sie als Eltern und Geschwister, wegfallen. 


\section{Deweys Verständnis von Demokratie und Bildung}

Es sollte deutlich geworden sein, dass es für einen gelingenden Umgang mit der Pandemie - gerade für sozial benachteiligte Menschen und solche mit Behinderungen - auch jenseits gerechter Gesundheitsversorgung gerechtere, sprich auch demokratischere und zukunftsorientierte Lösungen im Umgang mit gesellschaftlichen Krisensituationen braucht: zum einen, um keine Spiralen weiterer Benachteiligungen in Gang zu setzen, also Risiken cross-kategorial und invers-cross-kategorial zu verstärken. Jetzt schon zeigen sich nämlich Tendenzen, dass geschlossene Schulen und Home Schooling bestehende gesellschaftliche Vulnerabilitäten eher noch verstärken werden denn reduzieren (vgl. Schleicher 2020). Zum anderen ist es auch in epistemischer Hinsicht wichtig, existierende Marginalisierungen und Ungerechtigkeiten kulturell und sozial nicht weiter zu verstärken. Beides sind Aufgaben für alle Bürger*innen in einer Demokratie, nicht nur für deren politische Repräsentant*innen.

Das Modell einer Demokratie, die versucht, problematische Formen von Vulnerabilität zu vermeiden, entspricht einer inklusiven, diskursiven und partizipativen Demokratie (vgl. Young 2000). In einer solchen Demokratie fühlen sich alle Teilnehmenden dazu verpflichtet, Lösungen zu finden, die den Bedürfnissen und Interessen aller Bürger*innen gleichwertig gerecht werden, in der sich Menschen auch respektiert und in ihren (vielleicht auch gruppenspezifischen) Bedürfnissen ernst genommen fühlen. Denn nur in einem Kontext, in dem sich die Bürger*innen wechselseitig respektieren und sich um die Bedürfnisse und Interessen der anderen kümmern, besteht die Hoffnung, einen respektvollen, fürsorglichen Dialog zu etablieren, der zu einer wirklich kollaborativen und konsensuellen Entscheidungsfindung führt (vgl. Baker 2015, 82).

Eine solche Vorstellung von Demokratie schwebte dem US-amerikanischen Pragmatisten John Dewey vor, und anders als viele Denker*innen vor und nach ihm verknüpft er diese mit Bildung. Dewey entwarf konkret die Perspektive einer reflexiv-experimentellen Demokratie, die seiner Ansicht nach auch und bereits in der schulischen Bildung zum Ausdruck kommen sollte. Für Dewey (2000) spielte die Schule nämlich eine besondere Rolle bei der Entwicklung und Sicherung von Demokratien: Schulen könnten die Räume sein, in denen die Erfahrungen aller Lernenden sicht- und für alle hörbar gemacht, respektiert und zum Gegenstand von Kommunikationsprozessen werden, bei denen sowohl mitgeteilt als auch zugehört wird. Das bedingte eine aktive Beteiligung von Zuhörenden und Redenden, im übertragenen wie im wörtlichen Sinn. Richard Pring (2007, 119) formulierte dieses Verständnis Deweys wie folgt:

"Democracy is deep and active communication between individuals. It welcomes and sustains diversity of experience and background. It reflects the constant attempt to 
break down the barriers that inhibit communication - those of social class, racial stereotyping or selective schooling. Any such separateness impoverishes the experience of all. It blocks off the experiences of others from which one's own experience would be enriched."

Eine demokratische Schule wäre also nach Dewey eine Schule, in der die Bedürfnisse aller Lernenden aktiv Berücksichtigung finden, in der die Diversität der Erfahrungen einbezogen wird und Kommunikation wichtig ist.

Natürlich ist es in der aktuellen Situation - dieser Artikel ist im Februar 2021 geschrieben worden - die primäre Sorge, die medizinischen, gesundheitspolitischen und ökonomischen Begleiterscheinungen und Folgen der Pandemie zu bekämpfen und zu lindern. Mittel- und langfristig scheint es aber notwendig und angebracht, über die jeweiligen Maßnahmen, deren Beziehungen zueinander und die Art und Weise, wie über Behinderung und Menschen mit Behinderungen (nicht) gesprochen wird, kritisch zu reflektieren.

\section{Fazit}

Vulnerabilität, das zeigt nicht zuletzt der gesellschaftliche Umgang mit der aktuellen Covid-19-Pandemie, sollte nicht auf ihre anthropologische Komponente reduziert werden. Dabei würde nämlich ignoriert werden, dass einige Menschen (unter ihnen Menschen mit Behinderungen) vulnerabler sind als andere, nicht zuletzt (nochmals mit Pettit 2001 gesprochen) in sogenannt ,problematischer' Hinsicht. So haben Wolff und De-Shalit (2007) ausgeführt, dass die Risiken einer Behinderung nicht nur darin bestehen, dass sich Schädigungen und Funktionsbeeinträchtigungen verschlimmern, sondern auch darin, dass diese primären und vielleicht sogar gut behandel- respektive bewältigbaren Beeinträchtigungen auf andere Funktionen des Lebens übergreifen, oder dass beim Versuch, bestimmte Funktionen zu sichern, wiederum andere, dritte Funktionen gefährdet werden. Das gilt im besonderen Maße für Gesundheit, die über Kontexte wie Bildung oder Arbeit gefährdet werden kann und umgekehrt auch diese gefährdet.

Und auch in epistemischer Hinsicht zeigt sich, folgt man Frickers Theorie (2007), dass Menschen mit Behinderungen sowohl unter testimonialer (worauf ihre weitgehende Absenz von der medialen Berichterstattung über Covid-19 zumindest ein Hinweis ist) als auch unter hermeneutischer Ungerechtigkeit leiden. Von letzterem zeugen auch die Schwierigkeiten (vgl. Schulz 2020), dem Erlebten sprachlichen Ausdruck zu verleihen.

Schulen geraten in der ersten Phase einer solchen Krisenbewältigung vorwiegend als Risiko-Hotspots in den Fokus. Die Schule ist aber auch Praxisfeld für einen besseren Umgang mit gesellschaftlichen Herausforderungen, insofern die Schule 
ein Übungsfeld für aktives Zuhören wie auch aktives, dialogisches Mitreden ist. Das war die Idee, die Dewey für Schule hatte. Spätestens also für die Vorstellungen und Visionen zukünftiger Krisenbewältigung werden Schulen wichtig. Nicht nur, weil die Schule ein Ort ist, in welchem wichtige Weichen für eine gerechte und gleichberechtige gesellschaftliche Teilhabe gestellt werden, sondern auch, weil in ihr die dafür notwendigen kognitiven, motivationalen und handlungsorientierten Grundlagen für einen besseren Umgang mit (allen möglichen Arten von) gesellschaftlichen Krisen gelegt werden.

Covid-19 wird nicht die letzte globale Pandemie gewesen sein. Bei späteren Krisen werden diejenigen, die heute Kinder sind, Erwachsene sein, und einige von ihnen werden an entscheidenden Positionen sein, mit Macht, Kompetenz und Einfluss versehen, die entstehenden, unterschiedlich gelagerten Herausforderungen mitzugestalten. Die Hoffnung Deweys war es, dass demokratische Dialoge partizipativ, respektvoll und auf die Diversität der Bedürfnisse der Mitglieder einer Gesellschaft ausgerichteten gestaltet werden können. Schule ist dafür nicht nur ein Lern-, sondern auch ein Experimentierfeld.

\section{Literatur}

Baker, John (2015): Conceptions and Dimensions of Social Equality. In: Fourie, Carina, Schuppert, Fabian \& Wallimann-Helmer, Ivo (Hrsg.): Social Equality: On What It Means to Be Equal. Oxford, 65-86.

Dewey, John (2000): Demokratie und Erziehung - Eine Einleitung in die philosophische Pädagogik. 3. Aufl. Herausgegeben und mit einem Nachwort von Jürgen Oelkers. Basel und Weinheim.

Fricker, Miranda (2007): Epistemic Justice: Power and the Ethics of Knowing. Oxford.

Garrau, Marie \& Laborde, Cécile (2015): Relational Equality, Non-Domination, and Vulnerability. In: Fourie, Carina, Schuppert, Fabian \& Wallimann-Helmer, Ivo (Hrsg.): Social Equality: On What It Means to Be Equal. Oxford, 45-64.

Keller, Christoph (2020): „Wir Behinderten“ haben es nicht zum Thema gebracht. In: Tagesanzeiger, 5.5.2020. Online: https://www.tagesanzeiger.ch/wir-behinderten-haben-es-nicht-zum-themagebracht-131141068357 (Stand: 15.01.2021).

MacIntyre, Alasdair (1985): After Virtue: A Study in Moral Theory. London.

MacIntyre, Alasdair (1988): Whose Justice? Which Rationality? Notre Dame.

Nussbaum, Martha C. (2006): Frontiers of Justice: Disability, Nationality, Species Membership. Cambridge MA.

Pettit, Philip (2001): A Theory of Freedom: From the Psychology to the Politics of Agency. Oxford.

Pring, Richard (2007): John Dewey: A Philosopher of Education for Our Time? London.

Schulz, Sandra (2020): „Ich habe schon eine doppelte Lungenentzündung überlebt“. In: Der Spiegel, 10.04.2020. Online: https://www.spiegel.de/familie/coronavirus-menschen-mit-behinderungenin-der-coronakrise-a-e9706850-8067-4f74-829c-7a700d928a5e (Stand: 15.01.2021).

Schleicher, Andreas (2020): The Impact of Covid-19 on Education. Insights From Education at a Glance 2020. Paris.

Sen, Amartya (1999): Development as Freedom. Oxford.

Tronto, Joan C. (2013): Caring Democracy: Markets, Equality, and Justice. New York.

United Nations (2010): Factsheet on Persons with Disabilities. Genf.

Wolff, Jonathan \& De-Shalit, Avner (2007): Disadvantage. Oxford. 


\section{Franziska Felder}

World Health Organization (2020): Disability Considerations During the COVID-19 Outbreak. Online: https://www.who.int/publications/i/item/WHO-2019-nCoV-Disability-2020-1. (Stand: 15.01.2021).

World Health Organization: Weekly Epidemiological Update Coronavirus Disease (COVID-19) (5. Januar 2021). Online: https://www.who.int/publications/m/item/weekly-epidemiological-update--5-january-2021 (Stand: 03.03.2021).

Young, Iris Marion (2000): Inclusion and Democracy. Oxford. 